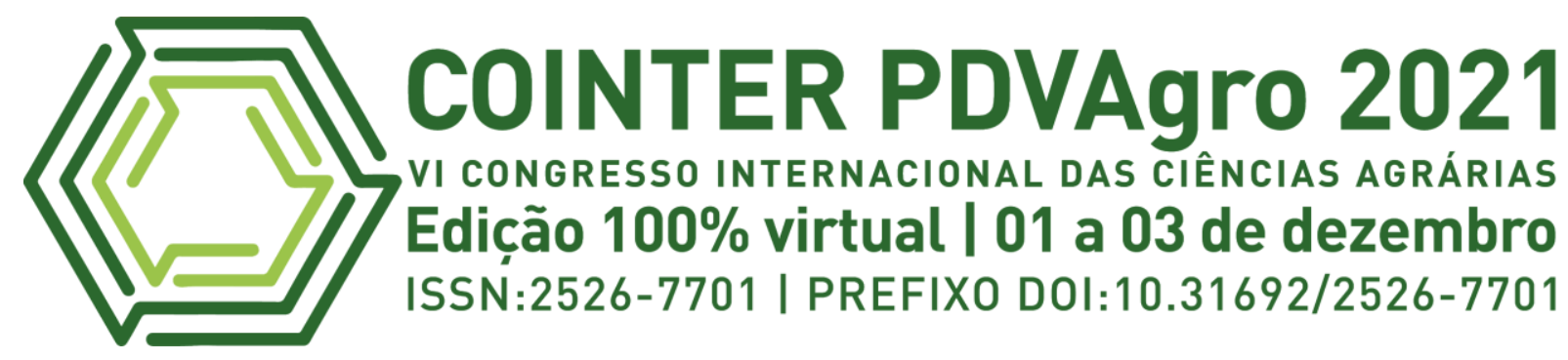

\title{
ANÁLISE DE REGRESSÃO LINEAR MÚLTIPLA: UMA APLICAÇÃO EM CAPIM ELEFANTE (P. Purpureum Schumach)
}

\section{ANÁLISIS DE REGRESIÓN LINEAL MÚLTIPLE: UNA APLICACIÓN EN HIERBA ELEFANTE (P. Purpureum Schumach)}

\section{MULTIPLE LINEAR REGRESSION ANALYSIS: AN APPLICATION IN ELEPHANT GRASS (P. Purpureum Schumach)}

\author{
Apresentação: Comunicação Oral \\ Adalberto Francisco da Silva Júnior ${ }^{1}$; Carén Beatriz dos Santos Felix da Silva ${ }^{2}$; Andréa Renilda Silva Soares ${ }^{3}$, \\ Jane Clésia Silva dos Santos; ${ }^{4 ;}$ Moacyr Cunha Filho ${ }^{5}$.
}

DOI: https://doi.org/10.31692/2526-7701.VICOINTERPDVAgro.0032

\section{RESUMO}

Neste trabalho são apresentados os resultados encontrados a partir da regressão linear múltipla utilizada no conjunto de dados proveniente de uma pesquisa sobre gramíneas forrageiras. O objetivo do presente estudo é ajustar dois modelos de regressão linear múltipla, no âmbito das ciências agrárias a partir de dados experimentais de variedades de capim elefante. Para isso, utilizou-se um conjunto de informações obtidas do experimento de campo, onde foram cultivadas cinco variedades de capim elefante (Cameroon, BRS, Capiaçu, Gramafante e Roxo). Contudo, buscou-se identificar a relação existente entre as variáveis independentes (prod. fresca e prod. seca) e a variável dependente (umidade). No modelo 1 , o coeficiente de determinação $\mathrm{R}^{2}$, que aplica a variação descrita pela equação gerada através da regressão linear múltipla, obtivemos 95,51\%, Para o modelo 2, após a realização da transformação logarítmica e montagem da planilha. Com as mesmas variáveis do modelo 1 , tanto a dependente quanto as independentes, não tiveram mudança. Ao calcular o coeficiente de determinação $\mathrm{R}^{2}$, foi de $99,64 \%$ das variações que podem ser explicadas pela equação e apenas $0,26 \%$ não são explicadas por elas, obtendo uma percentagem melhor que o modelo 1. Dentre os dois modelos de regressão linear múltipla analisado, constatamos que o resultado que melhor representou o problema proposto foi do modelo 2, pois foram utilizadas as mesmas variáveis pra ambos os modelos.

Palavras-Chave: Modelo estatístico; silagem; variáveis.

\section{RESUMEN}

En este trabajo se presentan los resultados encontrados a partir de la regresión lineal múltiple utilizada en el conjunto de datos de una investigación sobre pastos forrajeros. El objetivo del presente estudio es

\footnotetext{
${ }^{1}$ Graduando em Agronomia, bolsista PIBIC e estagiário Embrapa Solos, UFRPE-Recife, adalbertofrancisco75@gmail.com

${ }^{2}$ Mestranda em Biometria e Estatística Aplicada, bolsista CAPES, UFRPE-Recife, beatrizcaren310@gmail.com ${ }^{3}$ Mestranda em Biometria e Estatística Aplicada, bolsista CNPq, UFRPE- Recife, andrearenildaagronomia@gmail.com

${ }^{4}$ _Mestranda em Produção vegetal, UNIVASF, janeclesia28@gmail.com

${ }^{5}$ Pós Doutorado, UFRPE-Recife, moacyrcunha2006@ gmail.com
} 
ajustar dos modelos de regresión lineal múltiple, en el ámbito de las ciencias agrícolas, a partir de datos experimentales de variedades de pasto elefante. Para ello, se utilizó un conjunto de información obtenida en el experimento de campo, donde se cultivaron cinco variedades de pasto elefante (Camerún, BRS, Capiaçu, Gramafante y Roxo). Sin embargo, se buscó identificar la relación entre las variables independientes (rendimiento fresco y rendimiento seco) y la variable dependiente (humedad). En el modelo 1, el coeficiente de determinación $\mathrm{R}^{2}$, que aplica la variación descrita por la ecuación generada a través de la regresión lineal múltiple, obtuvimos un 95,51\%, Para el modelo 2, después de realizar la transformación logarítmica y montar la hoja de cálculo. Con las mismas variables que en el modelo 1, tanto las variables dependientes como las independientes no cambiaron. Al calcular el coeficiente de determinación $\mathrm{R}^{2}$, fue el $99,64 \%$ de las variaciones que pueden ser explicadas por la ecuación y sólo el $0,26 \%$ no son explicadas por ellas, obteniendo un mejor porcentaje que el modelo 1. Entre los dos modelos de regresión lineal múltiple analizados, encontramos que el resultado que mejor representaba el problema propuesto era el modelo 2, porque se utilizaron las mismas variables para ambos modelos.

Palabras clave: Modelo estadístico; ensilado; variables.

\begin{abstract}
In this paper the results found from the multiple linear regression used in the data set from a research on forage grasses are presented. The objective of the present study is to adjust two multiple linear regression models, in the field of agricultural sciences, based on experimental data of elephant grass varieties. For this, a set of information obtained from the field experiment was used, where five varieties of elephant grass (Cameroon, BRS, Capiaçu, Gramafante and Roxo) were grown. However, we sought to identify the relationship between the independent variables (fresh yield and dry yield) and the dependent variable (moisture). In model 1 , the coefficient of determination $\mathrm{R}^{2}$, which applies the variation described by the equation generated through multiple linear regression, we obtained $95.51 \%$, For model 2, after performing the logarithmic transformation and assembling the spreadsheet. With the same variables as in model 1 , both the dependent and independent variables did not change. When calculating the coefficient of determination $\mathrm{R}^{2}$, it was $99.64 \%$ of the variations that can be explained by the equation and only $0.26 \%$ are not explained by them, obtaining a better percentage than model 1. Among the two multiple linear regression models analyzed, we found that the result that best represented the proposed problem was model 2, because the same variables were used for both models.
\end{abstract}

Keywords: Statistical model; silage; variables.

\title{
INTRODUÇÃO
}

O Brasil apresenta uma estação seca bem definida acarretando uma baixa no fornecimento de forragens e influenciando negativamente na produção animal. Uma alternativa para amenizar tal problemática consiste no uso de técnicas que conservem as qualidades das forragens produzidas na estação chuvosa, tais como silagem, feno, forrageiras de inverno e outras (BARCELOS et al., 2018).

O capim elefante é uma excelente opção para a estabilidade, uma vez que esta gramínea apresenta robustez à escassez de água, tolerância a altas temperaturas, ciclo vegetativo curto, baixa exigência em fertilidade e capacidade de manter seu valor nutritivo mesmo com o avançar da sua maturidade, sendo possível a produção de silagem nestas condições, apresentando menor custo quando comparado a outras culturas como milho 
(QUINTINO et.al., 2016). Todavia, o capim elefante, visto que seu elevado teor de umidade acarreta em perdas na ensilagem, sendo uma desvantagem para a silagem (PINEDO et.al., 2019).

A regressão no âmbito da estatística pode ser compreendida como sendo o estudo da relação entre duas variáveis ou grupo de variáveis, onde se busca estimar o valor de uma variável a partir do conhecimento do valor de outra variável. Além de proporcionar estimar o grau de associação entre duas ou mais variáveis (SILVA; SILVA, 1982).

Segundo KRAJEWSKI, RITZMAN E MALHOTRA (2009), a regressão linear é um dos modelos causais mais conhecidos e utilizados, consiste de uma variável chamada dependente está relacionada com uma ou mais variaveis chamada de independentes por uma equação linear. Em uma linguagem técnica pode-se dizer que a linha de regressão minimiza os desvios quadrados dos dados reais (MEDEIROS E BIANCH, 2009).

A regressão pode ser expressa em dois modelos matemáticos. Caso se considere apenas uma variável independente é chamada de análise de regressão simples, caso seja duas ou mais variáveis, de análise de regressão múltipla (RODRIGUES, 2012).

A regressão simples define uma relação linear entre a variável dependente e uma variável independente. De acordo com SILVA (2016) este modelo trabalha com a utilização de uma variável explicativa $\mathrm{X}$ para a determinação do comportamento de uma variável $\mathrm{Y}$. Ou seja, uma relação linear entre a variavel dependente e uma variavel independente.

Enquanto tem-se uma regressão linear múltipla quando se admite que a variável resposta $Y$ é função de duas ou mais variavéis explicativas, $X_{1}, X_{2}, \ldots, X n$. Assim, o objetivo da análise de regressão múltipla é estabelecer uma equação que possa ser utilizada para predizer os valores de $Y$ considerando as diversas variaveis explicadas $X_{i}$.

O objetivo deste trabalho é ajustar dois modelos de regressão linear múltipla, no âmbito das ciências agrárias a partir de dados experimentais de variedades de capim elefante conforme expresso nas equações 1 e 2 .

\section{FUNDAMENTAÇÃO TEÓRICA}

\section{CAPIM ELEFANTE (P. Purpureum Schumach).}

As espécies do gênero Pennisetum se destacam entre as gramíneas forrageiras tropicais como uma alternativa em sistemas agrícolas tropicais, devido ao seu alto potencial produtivo e atributos agronômicos (MARANHÃO et al., 2020). Dentre o gênero, o capim elefante ( $P$. purpureum Schumach) se destaca devido o seu alto potencial produtivo associado como a qualidade, palatabilidade, vigor e persistência, o que têm induzido diversos programas de 
ANÁLISE DE REGRESSÃo LINEAR MÚLTIPLA:

melhoramento e cultivo pelo país (VIANA et al., 2018).

Apresenta como centro de origem a África e tem um alto índice de crescimento pode atingir uma produção de 40 toneladas de biomassa seca por hectare por ano (NAKANISHI et al., 2018). A sua grande variabilidade genética possibilita sua adaptação as mais diversas condições climáticas brasileiras. Sendo uma espécie considerada de alta eficiência fotossintética, o que contribui para uma grande capacidade de acúmulo de matéria seca e um alto percentual de fibra (SILVA et al., 2017). No estado de Pernambuco, o capim elefante tem amplo cultivo na forma de capineira, seja para fornecimento imediato aos animais ou para a produção de silagem. Sendo que há diverso cultivares disponíveis no mercado com características distintas, onde se destacam o Cameroon, BRS, Capiaçu, Gramafante e Roxo (PEREIRA et al., 2017).

O capim elefante cultivar Cameroon ( $P$. purpureum cv. Cameroon) se destaca por sua alta produção de biomassa e se adaptado à região do semiárido brasileiro, além de responderem bem à adubação orgânica (MENESES et al., 2018). O Capiaçu foi desenvolvido pela Embrapa e se destaca pelo porte ereto das touceiras com folhas largas e colmos grossos. Apresenta tolerância ao estresse hídrico e pode apresentar uma altura de 2,5 a 3,0 m aos 50-70 dias na estação das águas, podendo atingir uma produção de massa fresca de 54,3 e 93,4 t ha-1 aos 50 e 70 dias de idade de corte, respectivamente (PEREIRA et al., 2016).

A cultivar Gramafante pode atingir uma altura de $5 \mathrm{~m}$ de altura com touceiras densas. Além de alta rusticidade, fácil multiplicação e resistência considerável a seca e ao frio (OLIVEIRA, 2001). Já a cultivar roxo (P. purpureum cv. Roxo) se destaca por ter rizomas que possuem capacidade de armazenar água e solutos orgânicos, e também por possuir antocianinas nas lâminas foliares, que minimizam os efeitos deletérios da radiação solar excessiva e do déficit hídrico na assimilação de carbono (MARANHÃO et al., 2020).

\section{REGRESSÃO LINEAR MÚLTIPLA}

A regressão linear múltipla baseia-se na descrição de diferentes tipos de variáveis independentes, de maneira simultânea, sendo utilizada em análises empíricas, estatística, economia e demais ciências sociais aplicadas. Na regressão múltipla se considera situações que envolvem uma variedade de fatores para que ocorra a análise, quanto mais fatores tiverem para explicar uma variável, melhor será a análise empírica dos dados.

Segundo Sassi (2011), a regressão linear múltipla é uma análise multivariada que é utilizada em dados matemáticos entre duas variáveis em estudo, tendo por finalidade obter os valores pretendidos de uma variável dependente, permitindo a predição de resultados, por 
meio da correlação entre as variáveis independentes. A principal aplicação da regressão linear múltipla é na descrição de valores, baseado em um modelo matemático que descreve padrões de similaridade entre variáveis em estudo.

O modelo estatístico da regressão linear múltipla com $k$ variaveis regressoras $\left(X_{1}, X_{2}\right.$, ..., $X_{K}$ ) é dado por

$$
Y_{i}=\beta_{0}+\beta_{1} X_{i 1}+\beta_{2} X_{i 2}+\ldots+\beta_{k} X_{i K}+\varepsilon_{i}
$$

Em que:

- $Y_{i}$ é o valor observado de Y no i-ésimo nível de $\mathrm{X}$;

- $\beta_{0}, \beta_{1}, \beta_{2}, \ldots, \beta_{k}$ são parâmetros desconhecidos a serem estimados.

- $X_{i 1}, X_{i 2}, \ldots, X_{i K}$ é o i-ésimo nível das k variáveis independentes $(\mathrm{i}=1,2, \ldots, \mathrm{n})$.

- $\varepsilon_{i}$ é o erro que está associado à distância entre o valor observado $Y_{i}$ e o correspondente valor estimado $Y$, do modelo proposto, para o mesmo nível i de $X$.

O modelo de regressão linear para duas variáveis regressoras é

$$
Y_{i}=\beta_{0}+\beta_{1} X_{i 1}+\beta_{2} X_{i 2}+\varepsilon_{i}
$$

Para se obter a equação estimada, pode-se utilizar o método dos mínimos quadrados (MMQ), visando a minimização dos erros. Assim, tem-se que:

$$
\sum_{i=1}^{n} e_{i}^{2}=\sum_{i=1}^{n}\left[Y_{i}-\beta_{0}-\beta_{1} X_{i 1}-\beta_{2} X_{i 2}\right]^{2}
$$

Logo, os estimadores $\beta_{0}, \beta_{1}$ e $\beta_{2}$ que minimizam os erros é a solução do sistema de equações normais. De acordo com Sassi et al., (2021), quando se trabalha com análise de regressão, deve-se realizar a análise de variância (ANOVA) com o objetivo de comparar os modelos e testar a significância da regressão. Considerando o modelo de regressão linear múltipla, definido em (3), pode-se construir a tabela de análise de variância, corrigida pela

\begin{tabular}{|c|c|c|c|c|c|}
\hline & \multicolumn{5}{|c|}{$A N O V A$} \\
\hline & $\begin{array}{l}\text { Grau de } \\
\text { liberdade }\end{array}$ & $\begin{array}{c}\text { Soma de } \\
\text { Quadrados }\end{array}$ & $\begin{array}{c}\text { Soma de } \\
\text { Quadrados } \\
\text { Médios }\end{array}$ & $F_{\text {Calculado }}$ & $F_{\text {Tabelado }}$ \\
\hline Regressão & 2 & SQReg & $\frac{S Q R e g}{k}$ & $\frac{Q M \operatorname{Reg}}{Q M \operatorname{Res}}$ & $F_{\left(\alpha ; G L_{R e g}, G L_{r e s}\right)}$ \\
\hline Residual & $n-3$ & SQRes & $\frac{\text { SQRes }}{n-k-1}$ & & \\
\hline Total & $n-1$ & SQTotal & & & \\
\hline
\end{tabular}
média, dada por:

Tabela 01: Análise de variância.

Fonte: Sassi et al., (2021). 
O objetivo foi verificar a significância da regressão, assim as hipóteses para duas variáveis regressoras para o modelo linear múltiplo são:

$$
\begin{aligned}
& H_{0}: \beta_{1}=\beta_{2}=0 \\
H_{1}= & B_{j} \neq 0 \text { para qualquer } j=1,2 .
\end{aligned}
$$

Se rejeitarmos $\mathrm{H}_{0}$, temos que ao menos uma variável explicativa $X_{1}$ e $X_{2}$ contribue

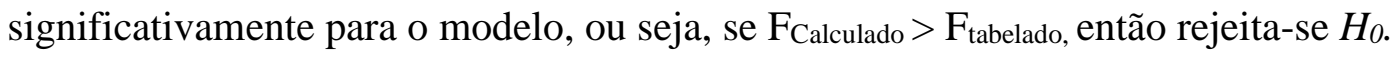

\section{METODOLOGIA}

Conduziu-se o experimento em campo, no período de janeiro a setembro de 2010, na Estação Experimental no município de Araripina (Sertão do Araripe) (Figura 01) no Instituto Agronômico de Pernambuco (IPA), localizada a 694 km do Recife, com coordenadas geográficas $07^{\circ} 27^{\prime} 37^{\prime}$ ' S e $40^{\circ} 24^{\prime} 36^{\prime}$ ' W e altitude de $831 \mathrm{~m}$, em um solo classificado como Latossolo Amarelo. Que de acordo com EMBRAPA (2011) são solos bastante uniformes em termos de cor, textura e estrutura; são profundos e muito profundos, bem drenados, com predominância de textura argilosa e muito argilosa.

Figura 01: Município de Araripina (Sertão do Araripe) - PE.

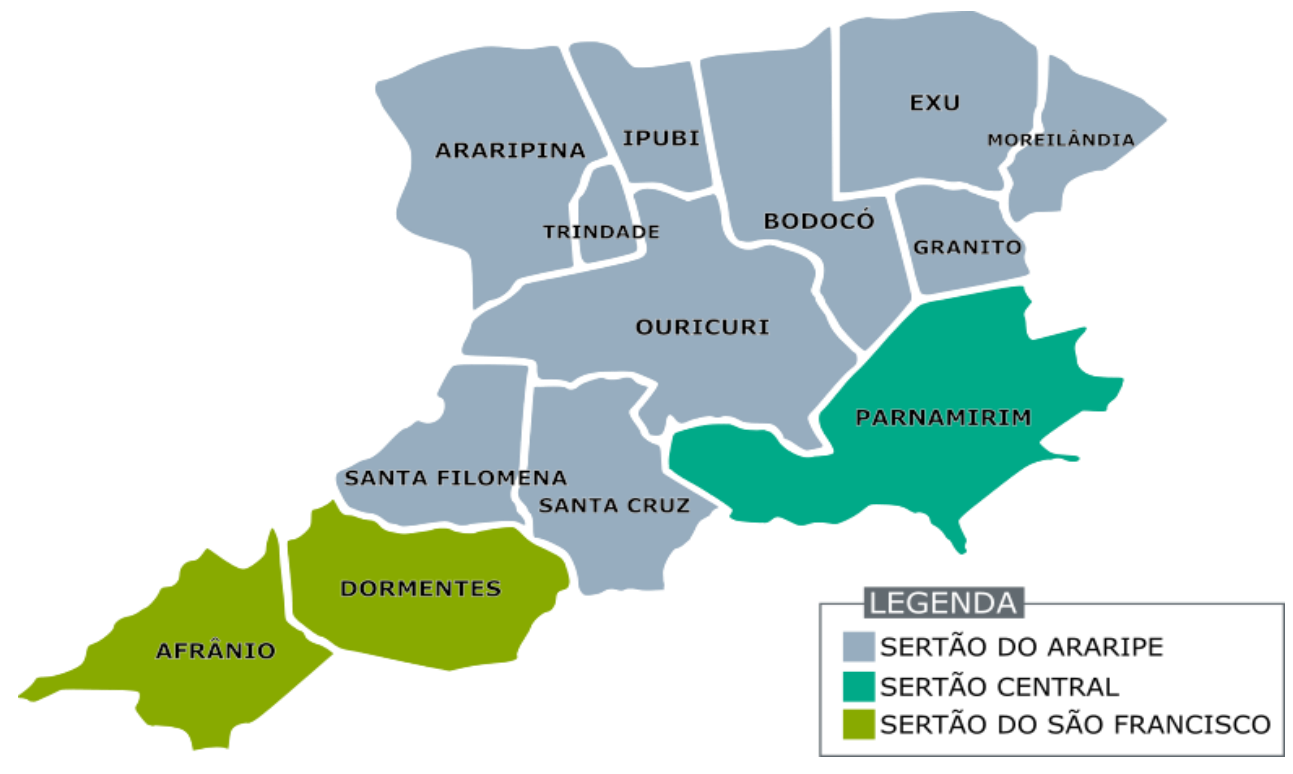

Fonte: CISAPE (2021).

A pesquisa foi de natureza qualitativa, que segundo SILVA (2019) é uma abordagem que possibilita ao pesquisador compreender detalhadamente os resultados. Foram cultivadas cinco variedades de capim elefante (Cameroon, BRS, Capiaçu, Gramafante e Roxo) durante 221 dias, e foram obtidos dados relacionados às variáveis na biomassa aérea. $\mathrm{O}$ experimento foi implantado em delineamento inteiramente casualizado. 
Considerando variáveis na biomassa aérea, umidade \%, produção de massa fresca (prod. fresca) e produção de massa seca (prod. seca), em kg/ha. Pode-se observar o conjunto de dados dessas variaveis na (Tabela 02). Neste trabalho, buscou-se identificar a relação existente entre as variáveis independentes (prod. fresca e prod. seca) e a variável dependente (umidade). Tornando umidade a variável resposta.

A fim de facilitar a regressão, sugere-se que as variáveis independentes estejam à direita da variável dependente. Ressaltando que as colunas parcela e variedades não são consideradas uma variável nesta situação.

Tabela 02: Planilha de dados com a variável dependente (umidade) e variaveis independentes (prod.

fresca e prod. seca)

\begin{tabular}{ccccc}
\hline PARCELA & VARIEDADE & UMIDADE \% $(\mathbf{Y})$ & $\begin{array}{c}\text { PROD. FRESCA } \\
\text { Kg/ha(X1) }\end{array}$ & $\begin{array}{c}\text { PROD. SECA } \\
\text { Kg/ha }(\mathbf{X 2})\end{array}$ \\
\hline $\mathbf{1}$ & & & 134904,58 & 44808,85 \\
$\mathbf{2}$ & BRS & 103,14 & 100293,34 & 38882,77 \\
$\mathbf{3}$ & CAMERON & 94,56 & 105636,96 & 35700,71 \\
$\mathbf{4}$ & GRAMAFANTE & 102,24 & 98035,42 & 38864,52 \\
$\mathbf{5}$ & ROXO & 93,22 & 60602,26 & 18866,99 \\
$\mathbf{6}$ & GRAMO & 106,36 & 77467,10 & 25856,79 \\
$\mathbf{7}$ & BRAFANTE & 102,89 & 89513,42 & 37341,23 \\
& & 90,01 & & \\
$\mathbf{8}$ & CAPIAÇU & 94,97 & 88957,44 & 34313,31 \\
$\mathbf{9}$ & CAPIAÇI & $91,192,837$ & 112845,29 & 46238,36 \\
$\mathbf{1 0}$ & CAMERON & 108,26 & 106425,53 & 42457,87 \\
$\mathbf{1 1}$ & CAPIACU & 103,72 & 58466,65 & 17482,96 \\
$\mathbf{1 2}$ & GRAMAFANTE & 91,63 & 78132,43 & 25660,64 \\
$\mathbf{1 3}$ & ROXO & 98,84 & 127474,47 & 51838,50 \\
$\mathbf{1 4}$ & BRS & 91,30 & 97959,44 & 35263,07 \\
$\mathbf{1 5}$ & CAMERON & 93,13 & 107099,82 & 43785,19 \\
$\mathbf{1 6}$ & GRAMAFANTE & 104,31 & 82899,38 & 32907,10 \\
$\mathbf{1 7}$ & CAPIAÇU & 98,32 & 71904,48 & 23340,19 \\
$\mathbf{1 8}$ & ROXO & 96,88 & 49729,68 & 18071,21 \\
$\mathbf{1 9}$ & BRS & 92,14 & 141775,92 & 52833,11 \\
$\mathbf{2 0}$ & CAMERON & 1949,82 & 108446,22 & 43742,98 \\
$\mathbf{T O T A L}$ & & 97,49 & 1898569,84 & 708256,36 \\
$\mathbf{M E ́} \mathbf{1}$ & & & 94928,49 & 35412,82 \\
\hline & & & & \\
\hline
\end{tabular}

Fonte: Própria (2021)

O ajuste dos modelos de regressão linear múltipla se deu através da equação 01, e a equação estimada através da equação 02, seguidas pelas etapas dos cálculos descritos na (Tabela 03).

Equação 01: Modelo linear múltipla analisada.

$$
Y_{i}=\beta_{0}+\beta_{1} X_{1}+\beta_{2} X_{2}+\varepsilon_{i}
$$




$$
Y_{i}=\beta_{0} X_{1}^{\beta_{1}} X_{2}^{\beta_{2}} \varepsilon_{i}
$$

Equação 02: Modelo linear da equação estimada por logaritimização.

$$
\ln Y_{i}=\ln \beta_{0}+\beta_{1} \ln X_{1}+\beta_{2} \ln X_{2}+\ln \varepsilon_{i}
$$

\begin{tabular}{|c|c|}
\hline 1 & $\begin{array}{l}\text { Inicialmente realizou o cálculo da correlação de Pearson, entre as variáveis a fim } \\
\text { de verificar a existência ou não da correlação; }\end{array}$ \\
\hline 2 & $\begin{array}{l}\text { Em seguida, deu-se início aos cálculos da regressão a fim de encontrarmos para } \\
\text { cada modelo, a equação que melhor explica as variáveis; }\end{array}$ \\
\hline 3 & $\begin{array}{l}\text { Calculou-se os desvios através do cálculo da soma dos quadrados e da soma dos } \\
\text { produtos, para se obter os coeficientes } b_{0}, b_{1}, b_{2} \text { e a partir de então, montar-se a } \\
\text { equação gerada pelo modelo; }\end{array}$ \\
\hline 4 & Logo após, realizou-se a análise de variância (ANOVA); \\
\hline 5 & $\begin{array}{l}\text { Além dos cálculos, do coeficiente de determinação }\left(\mathrm{R}^{2}\right) \text { e coeficiente de } \\
\text { determinação ajustado }\left(\mathrm{R}^{2}{ }^{2}\right) \text {; }\end{array}$ \\
\hline 6 & Análise gráfica dos resíduos; \\
\hline 7 & $\begin{array}{l}\text { Por fim, foi escolhiso a melhor equação para reaizar as contribuições de suas } \\
\text { variaveis independentes e assim verificar qual metodo explica melhor as } \\
\text { variaveis. }\end{array}$ \\
\hline
\end{tabular}

Tabela 03: Etapas de cálculo da regressão linear múltipla.

Fonte: Própria (2021).

\section{RESULTADOS E DISCUSSÃO}

Realizou-se o cálculo da correlação de Pearson, entre as variáveis a fim de verificar a existência ou não da correlação. Para o modelo 1 resultou numa correlação entre a variável dependente $Y$ (umidade) e variável independente $X_{1}$ (Prod. Fresca) de $-0,4$ e também entre variável dependente $Y$ (umidade) e variável independente $X_{2}$ (Prod. Seca) em -0,6. Observamos que ambas correlações se encaixam como correlação imperfeita negativa, devido estarem igual que $>-4$ e $<-1$.

A correlação teve o resultado em negativa, o que já era esperado, pois quando a prod. fresca e prod. seca aumentam, a umidade tende a diminuir, o que explica esta situação. 
Tabela 04: Resultado do calcúlo da analise de variância.

\begin{tabular}{lrcccc}
\hline \multicolumn{5}{c}{ ANOVA } \\
\hline & $G L$ & \multicolumn{1}{c}{$S Q$} & $Q M$ & $F_{\text {Calculado }}$ & $F_{\text {tabelado }}$ \\
Regressão & 2 & 609,4788049 & 304,7394 & 180,8517382 & $3,49359 \mathrm{E}-12$ \\
Residual & 17 & 28,64539701 & 1,685023 & & \\
Total & 19 & 638,1242019 & & & \\
\hline
\end{tabular}

Fonte: Própria (2021).

Como $\mathrm{F}_{\text {Calculado }}>\mathrm{F}_{\text {tabelado, }}$ podemos induzir que o modelo gerou uma equação altamente significativa.

Tabela 05: Resultado dos coeficientes, erro-padrão e valor $\mathrm{P}$ das variavéis.

\begin{tabular}{lccrr}
\hline & Coeficientes & \multicolumn{1}{c}{ Erro-padrão } & \multicolumn{1}{c}{ Stat $\boldsymbol{~}$} & \multicolumn{1}{c}{ valor $\boldsymbol{P}$} \\
\hline Interceptar & 100,8456149 & 1,247251337 & 80,85428 & $1,9912 \mathrm{E}-23$ \\
Variável $\boldsymbol{X}_{\mathbf{1}}$ & 0,000561188 & $4,17395 \mathrm{E}-05$ & 13,445 & $1,73465 \mathrm{E}-10$ \\
Variável $\boldsymbol{X}_{\mathbf{2}}$ & $-0,001599063$ & $9,54804 \mathrm{E}-05$ & $-16,7475$ & $5,32301 \mathrm{E}-12$ \\
\hline \multicolumn{5}{c}{ Fonte: Própria (2021). } \\
\end{tabular}

Ao calcular o coefficiente de determinação $\mathrm{R}^{2}$, que aplica a variação descrita pela equação gerada através da regressão linear múltipla, obtivemos 95,51\%, das variações que puderam ser explicadas pela equação, pode ser observada na Tabela 06, tendo apenas 4,49\% que não podem ser explicadas. Aplicando o $\mathrm{R}^{2}$ ajustado, também podemos observar que os valores das variações explicadas continuam altos com 94,98\%.

Tabela 06: Resultado das estatisticas de regressão.

\begin{tabular}{lc}
\hline Estatistica de regressão & \\
\hline $\mathbf{R}$ múltiplo & 0,977297292 \\
\hline $\mathbf{R}^{2}$ & 0,955109998 \\
$\mathbf{R}^{2}$ ajustado & 0,949828821 \\
Erro-padrão & 1,298084494 \\
Observações & 20 \\
\hline \multicolumn{2}{c}{} \\
\hline
\end{tabular}

A seguir está a representação gráfica das variáveis de prod. fresca $\left(X_{l}\right)$ e prod. seca $\left(X_{2}\right)$. 
Gráfico 01: Demonstração residual da variavél X1 (prod. fresca).

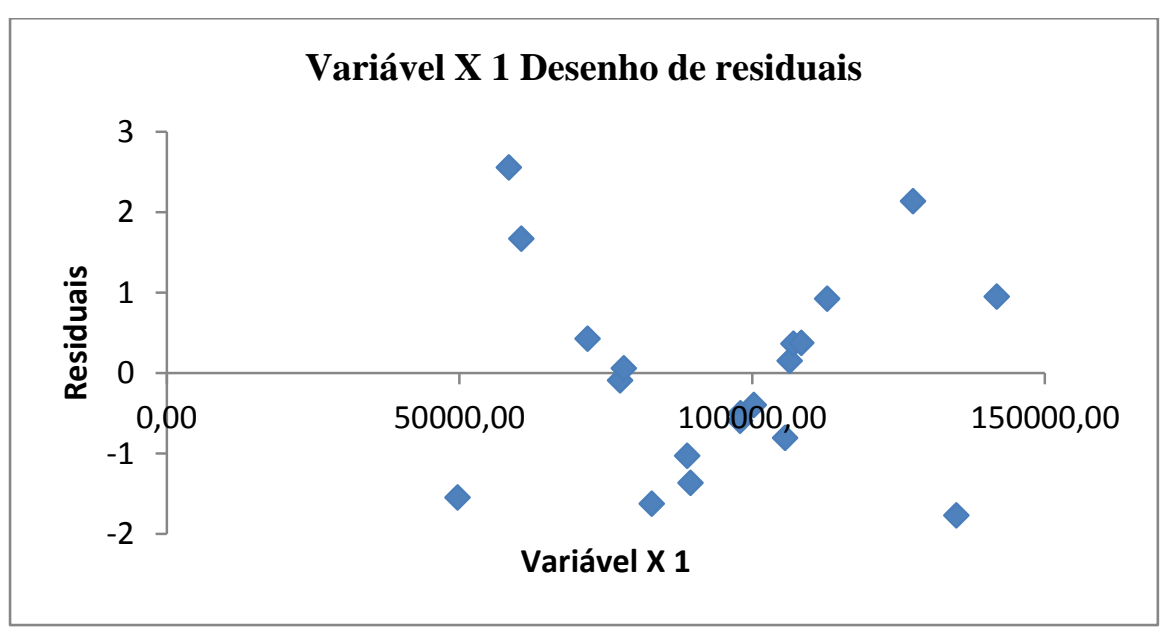

Fonte: Própria (2021)

Gráfico 02: Demonstração residual da variavel X2 (prod. seca).

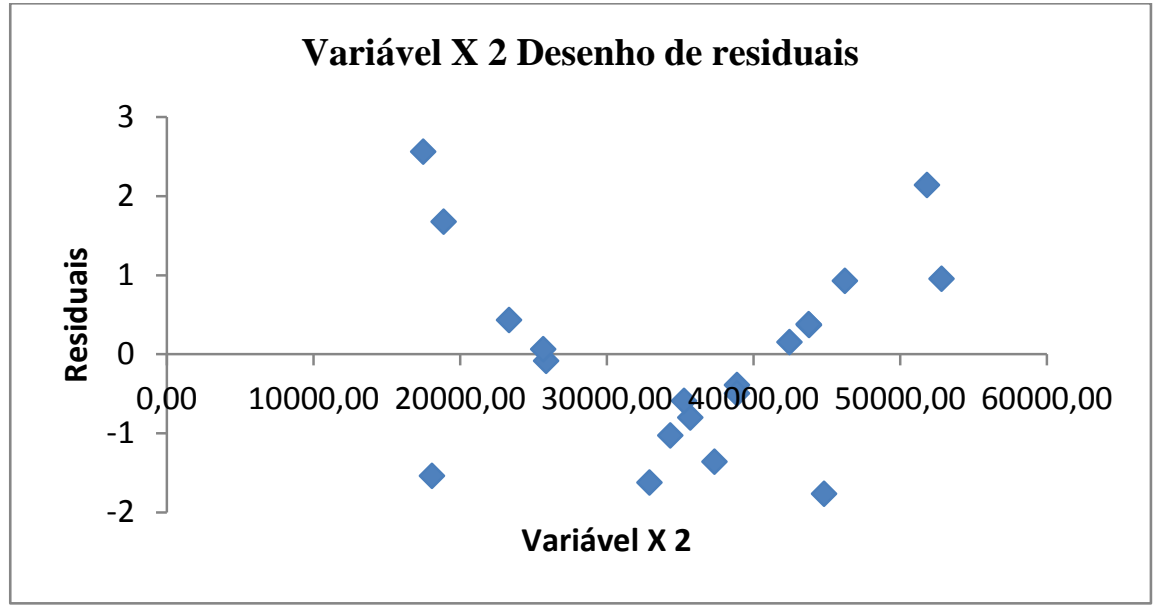

Fonte: Própria (2021).

Para o modelo 2, após a realização da transformação logarítmica e montagem da planilha. Com as mesmas variáveis do modelo 1, tanto a dependente quanto as independentes, não tiveram mudança. Desse moco, realizou-se o cálculo da correlação de Pearson, entre as variáveis a fim de verificar a existência ou não da correlação.

No qual resultou numa correlação entre a variável dependente $Y$ (umidade) e variável independente $X_{1}$ (Prod. Fresca) de $-0,5$ e também entre variável dependente $Y$ (umidade) e variável independente $X_{2}$ (Prod. Seca) em -0,7. Observamos que ambas correlações se encaixam como correlação imperfeita negativa, devido estarem $>-4<-1-$. A correlação também resultado em negativa, como explicado no modelo 1. Nas seguintes tabelas (Tabela 5), (Tabela 6) e (Tabela 7), podem-se observar os resultados do modelo 2. 
Tabela 07: Resultado do cálculo da análise de variância.

\begin{tabular}{lrrrrr}
\hline \multicolumn{5}{c}{$\boldsymbol{A N O V A}$} \\
\hline & $G L$ & \multicolumn{1}{c}{$S Q$} & $Q M$ & $F_{\text {Calculado }}$ & $F_{\text {tabelado }}$ \\
Regressão & 2 & 0,065697949 & 0,032849 & 2386,71226 & $1,49843 \mathrm{E}-21$ \\
Residual & 17 & 0,000233976 & $1,38 \mathrm{E}-05$ & & \\
Total & 19 & 0,065931925 & & & \\
\hline
\end{tabular}

Fonte: Própria (2021).

Podemos observar que $\mathrm{F}_{\text {Calculado }}>\mathrm{F}_{\text {tabelado, podemos afirmar também que o modelo }}$ gerou uma equação altamente significativa.

Tabela 08: Resultado dos coeficientes, erro-padrão e valor P das variavéis.

\begin{tabular}{lcccc}
\hline & Coeficientes & Erro-padrão & Stat t & valor $\boldsymbol{P}$ \\
\hline Interceptar & 3,973905237 & 0,046345805 & 85,74466 & $7,3545 \mathrm{E}-24$ \\
Variável X 1 & 0,570503405 & 0,01174567 & 48,57138 & $1,1106 \mathrm{E}-19$ \\
Variável X 2 & $-0,567421641$ & 0,009508068 & $-59,6779$ & $3,4171 \mathrm{E}-21$ \\
\hline \multicolumn{5}{c}{ Fonte: Própria (2021). }
\end{tabular}

Ao calcular o coeficente de determinação $\mathrm{R}^{2}$, podemos observar na tabela 08 , que 99,64\% das variações podem ser explciadas pela equação e apenas $0,26 \%$ não são explicadas por elas, obtendo uma porcentagem melhor que o modelo 1.

Ao calcular também o coeficiente de determinação ajustado, ou seja, $R_{a j}^{2}$, obtivemos o valor de $99,60 \%$ das variações explicadas pela equação e apenas $0,40 \%$ não são explicadas por ela.

Tabela 09: Resultado das estatísticas de regressão.

\begin{tabular}{lc}
\hline Estatistica de regressão & \\
\hline $\mathbf{R}$ múltiplo & 0,99822405 \\
$\mathbf{R}^{2}$ & 0,996451254 \\
$\mathbf{R}^{2}$ ajustado & 0,996033754 \\
Erro-padrão & 0,003709889 \\
Observações & 20 \\
\hline & Fonte: Própria (2021).
\end{tabular}

Em seguida, podem-se observar os gráficos residuais das variáveis de prod. fresca $\left(X_{l}\right)$ e prod. seca $\left(X_{2}\right)$. 
Gráfico 03: Demonstração residual da variável $X_{1}$ (prod. fresca).

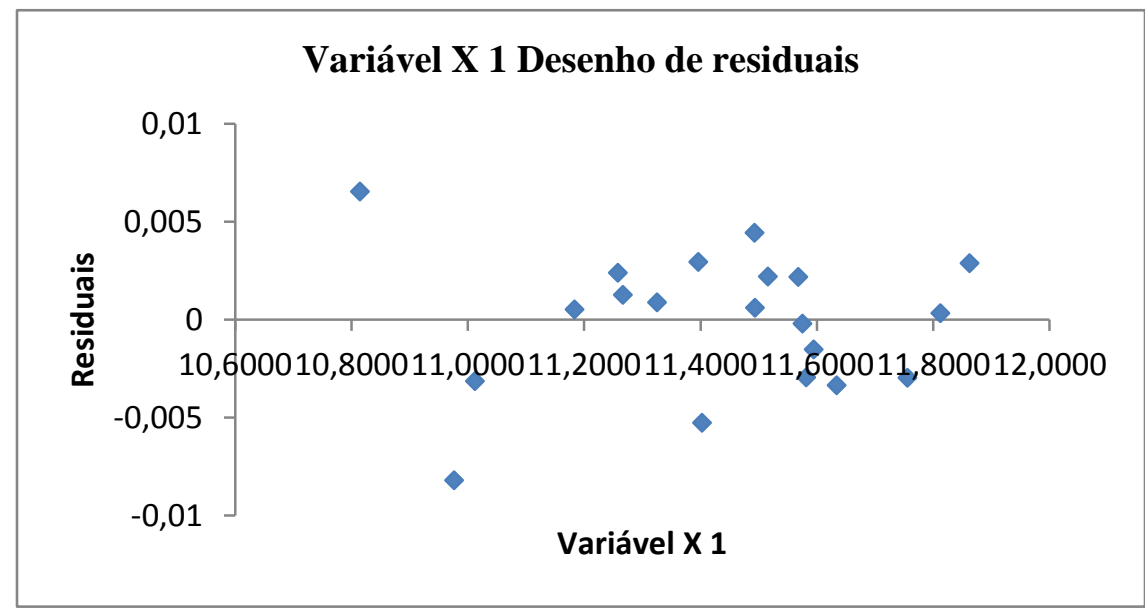

Fonte: Própria (2021).

Gráfico 04: Demonstração residual da variável $X_{2}$ (prod. seca).

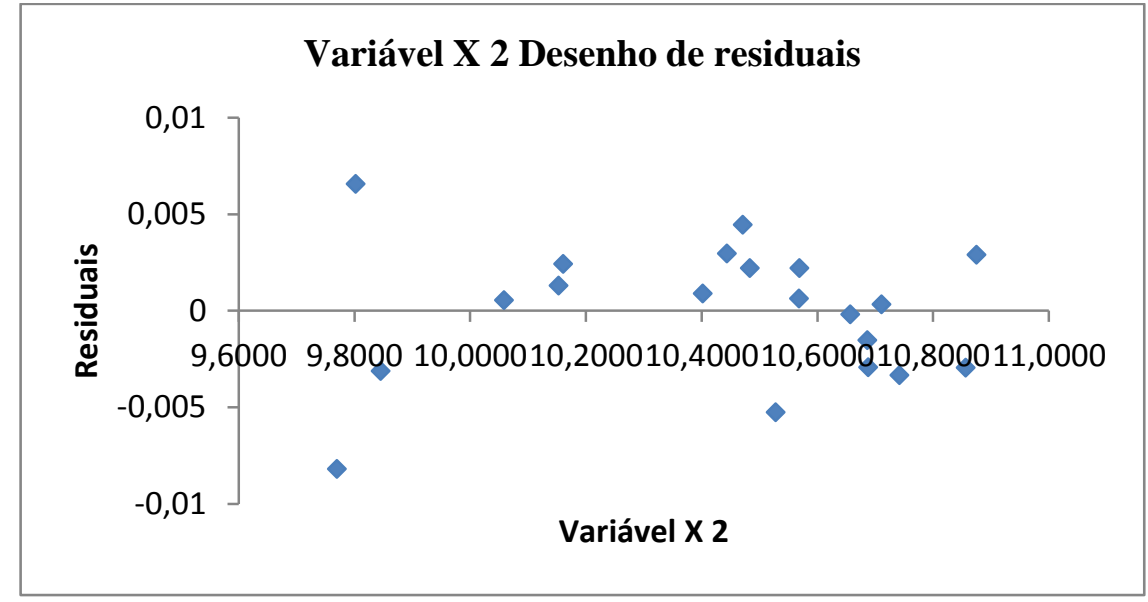

Fonte: Própria (2021).

Por fim, ao obter as estimadas $\beta_{0}, \beta_{1} e \beta_{2}$ foi calculada a equação estimada por logaritimização, no qual obtivemos a seguinte equação:

$$
\ln Y=3,973905+0,570503 \ln X_{1}-0,567421 \ln X_{2} .
$$

Desse modo, podemos concluir que dos dois modelos analisados, é perceptivel que os resultados do modelo 2 foram mais satisfatorio que o modelo 1, no qual teve 99,64\% das variações que podem ser explicadas pela equação, enquanto o modelo 1, teve 95,51\%. 


\section{CONCLUSÕES}

Dentre os dois modelos de regressão linear múltipla analisado, constatamos que o resultado que melhor respresentou o problema proposto foi do modelo 2, pois foram utilizadas as mesmas variaveis pra ambos os modelos. Mesmo assim, o modelo 2 obteve o melhor $\mathrm{R}^{2}$ ajustado, com 99,60\% das variações que podem ser explicadas pela equação, enquanto no modelo 1 foi de $94,98 \%$.

Podemos concluir que, conforme a produção de massa fresca e a produção de massa seca aumentam, a umidade tende a diminuir.

\section{REFERÊNCIAS}

BARCELOS, A. F.; CARVALHO, J. R. R. de; TAVARES, V. B.; GONÇALVES, C. C. de M. Valor Nutritivo e Características Fermentativas da Silagem de Capim-Elefante com Diferentes Proporções de Casca de Café. Ciência Animal Brasileira, Goiânia, v. 19, p. 1-12, 2018.

EMBRAPA, Empresa Brasileira de Pesquisa Agropecuária. Solos- caracterização e desafios tecnológicos, 2011.

FARIA，D.J.G; GARCIA，R.; TONUCCI，R.G.; TAVARES，V.B.; PEREIRA，O.G.; FONSECA, D. M. Produção e composição do efluente da silagem de capim-elefante com casca de café. Revista Brasileira de Zootecnia. 2010; 39(3):471- 78.Viçosa.

FRIAS, M.; SAVASTANO JUNIOR, H. Efeito do Tratamento de Água Quente nas Cinzas de Capim Elefante Calcinadas em Diferentes Preços. Revista Matéria, Rio de Janeiro, v. 23, n. 3, 2018.

KRAJEWSKI, L. J.; RITZMAN, L. P.; MALHOTRA, M. Administração da produção e operações. 8. ed. São Paulo: Pearson Prentice Hall, 2009.

MARANHÃO, T. D.; LOPES, M. N.; ALVES, F. G. da S.; SILVA, R. R. da; POMPEU, R. C. F. F.; SOUZA, W. do N.; LACERDA, G. F. Índices de Crescimento do Pennisetum purpureum cv. Roxo Cultivado em Diferentes Épocas sob Regime de Sequeiro. Revista Brasileira de Saúde e Produção Animal, Salvador, v. 21, p. 01 -14, 2020.

MEDEIROS, F. S. B.; BIANCHI, R. C. THE APPLICATION OF THE METHOD IN LINEAR REGRESSION DEMAND SEASONAL PRODUCTS: A CASE STUDY. Disciplinarum Scientia. Série: Ciências Sociais Aplicadas, S. Maria, v. 5, n. 1, p. 35-53, 2009. ISSN 1981-5778.

MENESES, A. J. G.; CÂNDIDO, M. J. D.; POMPEU, R. C. F. F.; SOUZA, H. A. de; GUEDES, F. L. Morfogênese do Capim-elefante Adubado com Composto Orgânico de Resíduos Sólidos em Pequenos Ruminantes. Revista Ciência Agronômica, Fortaleza, v. 49, n. 4, p. $699-707,2018$. 
NAKANISHI, E. Y.; SANTOS, V. dos; CABRAL, M. R.; SANTOS, S. F.; RODRIGUES, M. S.; OLIVEIRA, M. C. Gramafante: uma nova cultivar de capim elefante para o Vale do São Francisco. Embrapa Semiárido. Boletim de Pesquisa e Desenvolvimento, 14p, 2001.

PEREIRA, A. V; LEDO, F. J. S; MORENZ, M. J. F; LEITE, J. L. B; SANTOS, A. M. B; MARTINS, C. E; MACHADO, J. C. BRS Capiaçu: cultivar de capim-elefante de alto rendimento para produção de silagem. Embrapa Gado de Leite-Comunicado Técnico (INFOTECA-E), 2016.

PEREIRA, A.V.; LÉDO, F. J. S.; MACHADO, J. C; BARBOSA, S. BRS Kurumi and BRS Capiaçu - New elephant grass cultivars for grazing and cut-and-carry system. Crop Breeding and Applied Biotechnology, Viçosa, v. 17, p. 59-62, 2017.

PINEDO,L.A.SANTOS,B.R.C.,FIRMINO,S.S.,ASSIS,L.C.S.L.C.,BRAGA,A.P.,LIMA,P.O., OLIVEIRA,P.V.C.,\&PINTO,M.M.F.(2019). Silagem de sorgo aditivada com coproduto alternativo da torta de semente de cupuaçu. Brazilian Journ of Development, 12 (5), 2963329645.

QUINTINO,A.DAC.,ABREU,J.G.,ALMEIDA,R.G.,MACEDO,M.C.M.,CABRA,L.DAS.,\&G ALATI,R.L.(2016). Valor nutritivo de silagem de capim-piatã em monocultivo e em consórcio com sorgo de corte e pastejo. Ciência Animal Brasileira, 17 (2), 185-193.

RODRIGUES, s. A. Modelo de Regressão Linear e suas Aplicações. Universidade da Beira Interior Ciências. 2012.

SASSI, C. P. et al. Modelo de regressão linear múltipla utilizando os softwares R e Statistica: uma aplicação a dados de conservação de frutas. [2011?]. Disponível em: https://www.fc.unesp.br/Home/Departamentos/Matematica/revistacqd2228/v07a10-regressaolinear-multipla.pdf . Acesso em: 29 ago. 2021.

SILVA, C. B. S. F. S. Jogos como recursos pedagógicos no ensino: um levantamento sobre o que pensam os professores de matemática. Anais IV CONAPESC... Campina Grande: Realize Editora, 2019.

SILVA, J. A. A. da; SILVA, I. P. da. Estatística Experimental Aplicada à Ciência Florestal. Recife: UFRPE, 1982.

SILVA, J. P. C. Modelos de Regressão Linear e Logistica utilizando o software R. Dissertação (Mestrado em Estatística, Matemática e Computação), Universidade Aberta, Lisboa: UAB, 2016.

SILVA, V. B. da; DAHER, R. F.; MENEZES, B. R. da S.; OLIVEIRA, M. L. F.; ARAÚJO, M. do S. B.; NOVO A. A. C. Métodos de Estabilidade Diferentes para Recomendação de Cultivar em Capim Elefante para Fins de Energia no Brasil. Cerne, Lavras, v. 23, n. 4, p. $507-$ $515,2017$.

VIANA, B. L.; MELLO, A. C. L. de; GUIM, A.; LIRA, M. de A.; DUBEUX JÚNIOR, J. C. B.; SANTOS, M. V. F.; CUNHA, M. V. da. Características Morfológicas e Proporção de Tecidos da Lâmina Foliar em Clones de Capim Elefante sob Pastejo de Ovelhas. Pesquisa Agropecuária Brasileira, Brasília, v. 53, n. 11, p. 1268-1275, 2018. 